\title{
Manipulating the soil microbiomes during a community recovery process with plant beneficial species for the suppression of Fusarium wilt of watermelon
}

\author{
Xu Zhang ${ }^{\dagger}$, Chao Xue ${ }^{\dagger}$, Dan Fang, Xiaohui He, Mengyu Wei, Chenjin Zhuo, Junyao Jin, Biao Shen, Rong Li, \\ Ning Ling and Qirong Shen*
}

\begin{abstract}
Fusarium wilt is a devastating disease which impacts watermelon production. Soil fumigation using dazomet followed by biological organic fertilizer was applied to suppress the Fusarium wilt disease. We propose that fumigation suppresses the soil indigenous community, especially the soil-borne pathogens, while the utilization of bio-organic fertilizer facilitates the recovery of the soil microbiome to a beneficial, suppressive state through the introduction of plant growth-promoting microorganisms. Greenhouse experiment showed that applied biological organic fertilizer after dazomet fumigation effective restrain the disease incidence with a 93.6\% disease control. Fumigation strongly decreased soil microbial diversity and altered relative taxa abundances, suggesting the possibility of niche release by the resident soil microbial community. Fumigation followed by bio-fertilizer transformed the soil microbial community composition and resulted in higher relative abundances of beneficial microbial groups such as Bacillus (8.5\%) and Trichoderma (13.5\%), coupled with lower Fusarium abundance compared to other treatments. Network analysis illustrated that soil fumigation decreased interactions within the soil microbial community with less nodes and links while bio-fertilizer addition promoted node interactions. In addition, bio-fertilizer addition after fumigation resulted in the beneficial species becoming the key network connectors. Collectively, fumigation appears to release the resident soil niche resulting in lower diversity while the beneficial microbes introduced by bio-fertilizer addition colonize these niches, leading to a more complex community with fewer pathogens that suppresses Fusarium wilt disease incidence.
\end{abstract}

Keywords: Watermelon Fusarium wilt disease, Soil microbial resistance and resilience, Dazomet fumigation,

Biological organic fertilizer, Soil microbial assembly

\section{Introduction}

Watermelon Fusarium wilt as a serious plant disease worldwide was arose from the pathogenic fungus Fusarium oxysporum f. sp. niveum (FON) (Ling et al. 2010a). which seriously restrains watermelon production

\footnotetext{
*Correspondence: shenqr0203@163.com

†Xu Zhang and Chao Xue contributed equally to this work

Jiangsu Provincial Key Lab for Solid Organic Waste Utilization, National

Engineering Research Center for Organic-Based Fertilizers, Jiangsu

Collaborative Innovation Center for Solid Organic Waste Resource

Utilization, Nanjing Agricultural University, Nanjing 210095, China
}

worldwide (Zhou and Everts 2004). Dazomet (tetrahydro-3,5-dimethyl-2H-1,3,5-thiadiazine-2-thione) is often employed to suppress serious Fusarium infection in watermelon (Slusarski and Pietr 2009; Tian et al. 2014). When dazomet is applied to moist and hyperthermal soils, the fumigant decomposes into methyl isothiocyanate (MITC), which can effectively suppress fungi, nematodes, and weeds (Saeed et al. 2007). Many beneficial antergic species, for instance, Bacillus spp., and Trichoderma spp., which isolated from suppressive soil were utilized for the suppression of Fusarium wilt disease
Springer Open

(c) The Author(s) 2021. This article is licensed under a Creative Commons Attribution 4.0 International License, which permits use, sharing, adaptation, distribution and reproduction in any medium or format, as long as you give appropriate credit to the original author(s) and the source, provide a link to the Creative Commons licence, and indicate if changes were made. The images or other third party material in this article are included in the article's Creative Commons licence, unless indicated otherwise in a credit line to the material. If material is not included in the article's Creative Commons licence and your intended use is not permitted by statutory regulation or exceeds the permitted use, you will need to obtain permission directly from the copyright holder. To view a copy of this licence, visit http://creativeco mmons.org/licenses/by/4.0/. 
already (La Fuente et al. 2006; Qiu et al. 2012; Yuan et al. 2016). Beneficial species premixed with compost and amino acid as effective fertilizers in the suppression of many cash crops soil-borne disease have been widely reported (Luo et al. 2010; Qiu et al. 2012; Wu et al. 2014). The extra spaces and nutrients provided by bio-organic amendments for the antergic species that facilitates their colonization and pathogen antagonism (Xiong et al. 2017a). Such fertilizers increase the soil fertility and reduce the adverse impact of disease pathogens, thereby improve the plant vitality (Bailey and Lazarovits 2003). Nevertheless, few is understood concerning the underlying mechanisms of fumigation using dazomet followed by biological organic fertilizer amendment during the process of soil-borne disease inhibition.

Soil microbes have a determinate place within the sustainability of soil biological activity, maintaining ecological balance for the soil ecosystem and plant production through their metabolic activities and energy exchanges (Zarraonaindia et al. 2015). It is necessary to understand the contribution of soil microbial community resistance and resilience during the manipulation of the resident soil microflora for the disease suppression (Allison and Martiny 2008). A outside disturbance is often caused by biotic or abiotic elements, which leads to a microbial community tiny destabilization or a 'stress', such as the reaction from the independent or the whole ecological system. On the basis of their length of time, disturbances are usually divided into pulses and presses, which affect the soil physicochemical properties (Rykiel 1985). In general, fumigation can be recognized as a 'disturbance', which is a pulse disturbance as it is a short-term event. Dazomet fumigation may exert a toxic impact on the microbial community due to the methyl-isothiocyanate and sulphuric acid it volatilizes which negatively effects soil microorganisms, including the beneficial species (Scopa and Dumontet 2007). Ultimately, the recovery of soil productivity is dependent on the re-establishment of the soil microbial activity and the creation of a biological environment for soil diseases control (Eo and Park 2014). In consideration of the decisive importance of maintaining soil microbial ecosystems for long-term productivity, large numbers of vigor on realizing the reactivity (including resistance and resilience) of microbial community to extraneous disturbances has been put into effect (Wertz et al. 2007; Fujino et al. 2008). Fumigation followed by the biological organic fertilizer application amendment is able to suppress soil-borne wilt disease through decreasing the number of pathogens and by modifying the resident soil microbial community diversity and/or structure.

Our hypothesis is that soil fumigation will overcome the inherent resistance of microbial community also suppress the resident soil community, including both pathogens and beneficial species. Beneficial microbiota introduced through bio-fertilizer application will further suppress pathogen growth while promoting crop viability through manipulating the resident microbial community during the period of community resilience. To test our hypothesis, greenhouse experiment was carried out to confirm the effectiveness of the biological organic fertilizer application after dazomet fumigation on the control of watermelon Fusarium wilt disease. The soil samples were gathered, and soil microbial composition and diversity were accessed after soil fumigation and biofertilizer application. Microbial ecological network was established with the purpose of exploring community interaction and complexity to reveal the effect of an integrated agricultural strategy on soil microflora.

\section{Materials and methods}

\section{Microorganisms, organic and biological organic fertilizer preparation}

Antagonistic bacterium Bacillus amyloliquefaciens SQR9 (CGMCC NO.5808, China Microbial Culture Collection Committee General Microbiology Center) and the fungal strain Trichoderma guizhouense NJAU4742 (CGMCC NO.12166, China Microbial Culture Collection Committee General Microbiology Center) were separated by the Jiangsu Provincial Key Lab for Organic Solid Waste Utilization, Nanjing Agricultural University. Both strains showed negative impact on the fungal pathogen F. oxysporum growth (Cao et al. 2011; Yuan et al. 2016). Bio-fertilizer preparation process was described previously, at a word, a certain number of antagonistic strain SQR9 and NJAU4742 were added into compost for second solid fermentation and finally reach the concentration of $10^{9} \mathrm{CFU} \mathrm{g}^{-1}$ and $10^{8} \mathrm{CFU} \mathrm{g}^{-1}$, respectively (Ling et al. 2010b; Zhang et al. 2008).

\section{Field experiment}

Field experiment was conducted in Huaian $\left(33^{\circ} 35^{\prime} \mathrm{N}\right.$, $119^{\circ} 01^{\prime}$ E), Jiangsu Province, China from March to July 2017. The average temperature in the greenhouse was $25{ }^{\circ} \mathrm{C}$ during the whole process of watermelon growth. Watermelon was continuously cultivated in the greenhouse for more than 4 years (eight growing seasons). The Fusarium wilt disease incidence reached up to $80 \%$ with approximately $10^{5} \mathrm{CFU}$ of FON per gram soil in the previous growing season. The soil has a $\mathrm{pH}$ value of 6.15 , a total nitrogen content of $0.62 \mathrm{~g} \mathrm{~kg}^{-1}$ and available phosphorus, potassium contents of $60,256 \mathrm{mg} \mathrm{kg}^{-1}$ respectively.

Two treatments (FOF, FBOF) and a control (OF) were set to test the effect of dazomet fumigation followed by biological organic fertilizer on watermelon Fusarium wilt disease: (I) Soil fumigated with dazomet (375 kg per ha) 
and treated with biological organic fertilizer $(7500 \mathrm{~kg}$ per ha) was assigned as FBOF; (II) Soil treated with organic fertilizer (7500 kg per ha) following fumigation with dazomet (375 kg per ha) was assigned as FOF. (III) Soil treated with organic fertilizer (7500 kg per ha) without fumigation served as control (OF). Bio-organic fertilizer is a mixture of organic fertilizer and agricultural amino acid inoculated with Bacillus amyloliquefaciens SQR9 and Trichoderma guizhouense NJAU4742 which were separated by our laboratory to suppress soil-borne diseases. Nutrients in the control and two treatments were equal.

The dazomet was applied into the soil evenly after soil was tilled completely. Then the soil was covered by plastic film after watered to achieve $40 \%$ moisture. The plastic film was took away after 10 days, which meant fumigation was terminated. Fertilizers were added to the soil after another 7 days followed by another tillage. Watermelon seedlings (Sumeng No.6) which grown to two tender buds were transplanted into the soil. Eight replicates $\left(5 \mathrm{~m}^{2}\right.$ for each) with 10 seedlings were set in each treatment. Watermelon seeds were first soaked in warm water at $60{ }^{\circ} \mathrm{C}$, stirred to room temperature, sterilized with $5 \%$ sodium hypochlorite for $3 \mathrm{~min}$, then germinated in incubator after washed with sterile water for 3-4 times, and transplanted after they grow to two leaves.

After harvested, plants which exerted Fusarium wilt disease symptoms were counted, then soil samples were collected. In this study, the percentage of diseased plants to the total plants was computed to express the morbidity.

\section{Soil sampling and total DNA extraction}

Soil from the top $15 \mathrm{~cm}$ was sampled used five-point sampling method after watermelons were harvested. Briefly, soil from five different holes were collected and mixed as one soil sample of a plot. Soil samples collected from each treatment were placed into ice box for retaining freshness then transported to the laboratory. Finally, soil total DNA was extracted under the manufacturer's protocol of the Power soil DNA Extraction kit (MOBIO Laboratories, 108 Carlsbad, CA, USA).

\section{Quantification of pathogens}

Fusarium oxysporum selective medium was used for plate counting which one liter containing $1 \mathrm{~g} \mathrm{~K}_{2} \mathrm{HPO}_{4}, 0.5 \mathrm{~g}$ $\mathrm{KCl}, 0.5 \mathrm{~g} \mathrm{MgSO}_{4}, 0.01 \mathrm{~g}$ Fe-Na-EDTA, $2 \mathrm{~g}$ L-asparagin, $20 \mathrm{~g}$ D-galactose and $1000 \mathrm{~mL}$ water (Komada 1975). $5 \mathrm{~g}$ of soil in each sample was added to a $150 \mathrm{~mL}$ conical flask containing $45 \mathrm{~mL}$ of sterilized distilled water. Next, the soil suspension was diluted and applied on the medium after vibrating for $30 \mathrm{~min}$ at $170 \mathrm{rpm}$ under room temperature on the shaker.

\section{Soil total DNA amplification and sequencing}

Primer set 338F (5'-ACTCCTACGGGAGGCAGC AG-3') and 806R (5'-GGACTACHVGGGTWTCTAAT$3^{\prime}$ ) (Xu et al. 2016) were utilized for the amplification of the 16S rRNA V3-V4 variable regions. ITS3F (5'-GCA TCGATGAAGAACGCAGC-3') and ITS4R (5'- TCC TCCGCTTATTGATATGC-3') (White et al. 1990) were utilized for the amplification of the fungal internal transcribed spacer (ITS). All the amplifications were conducted in a $50 \mu \mathrm{L}$ mixture including $2 \mu \mathrm{L}$ DNA template, $10 \mu \mathrm{L} 5 \times \mathrm{Q} 5$ reaction buffer, $4 \mu \mathrm{L}$ of $2.5 \mathrm{mM}$ dNTPs, $2.5 \mu \mathrm{L}$ of each primer and $0.5 \mu \mathrm{L}$ Q5 high-fidelity DNA polymerase (New England Biolabs, UK) using Eppendorf Mastercycler nexus gradient (Eppendorf AG, Hamburg, Gemany). The conditions of Polymerase Chain Reaction (PCR) were $98^{\circ} \mathrm{C}$ for $2 \mathrm{~min}$, and then 35 cycles of $10 \mathrm{~s}$ at $98^{\circ} \mathrm{C}, 15$ s under $59{ }^{\circ} \mathrm{C}, 30 \mathrm{~s}$ at $72{ }^{\circ} \mathrm{C}, 72^{\circ} \mathrm{C}$ for 2 min, followed by storage at $10^{\circ} \mathrm{C}$.

\section{Bioinformatics analysis}

Sequence analyses were mainly accomplished with QIIME (Quantitative Insights Into Microbial Ecology) pipeline (Caporaso et al. 2010). Briefly, raw paired-end sequences were assembled, and then low quality $(\mathrm{q}>0.5)$ and short reads $(\mathrm{bp}<200)$ were filtered. The assembled OTUs were clustered at $3 \%$ dissimilarity and OTU tables were performed. Alpha diversity concluding Chao and Shannon indices were calculated under the OTU table. Non-Metric Multidimensional Scaling (NMDS) analysis based on Bray-Curtis distances was figured up using vegan packages in $\mathrm{R}$ (version 3.3.0). Manhattan plots were carried out to explore the differences between the control and treatments at the phyla level using edgeR and dplyr packages in $\mathrm{R}$ (version 3.3.0). Raw sequences from each sample were uploaded to the NCBI Sequence Read Archive (SRA) database (Accession Number: SUB4843217).

\section{Network analysis}

Phylogenetic molecular ecological networks (pMENs) of different treatments were performed on the basis of the Molecular Ecological Network Analyses Pipeline (MENAP) (http://129.15.40.240/mena/) (Deng et al. 2012; Zhou et al. 2010). First, a matrix of OTU-based microbial variables table file was formed as the formats required. Then, a pairwise Pearson correlation between any two OTUs was calculated by an RMT-based measure based on the abundance data. The network characteristics were determined by module detection calculations. Finally, the network graph was visualized by Gephi platform (Ling et al. 2016; Zhou et al. 2010, 2011). Zi and Pi 
values were calculated to represent the topological roles of each nodes and the threshold of them for classifying these OTUs were 2.5 and 0.62 , respectively.

\section{Statistical analysis}

Multiple comparisons between samples were carried out utilizing a one-way ANOVA (Duncan test) with significance indicated at $P<0.05$ using IBM SPSS 19.0 (IBM Corporation, New York, United States). Mantel tests and pearson correlation analysis between microbial relative abundances and morbidity were performed using $\mathrm{R}$ (version 3.3.0).

\section{Results}

\section{Disease incidence and quantification of pathogens}

The watermelon Fusarium wilt incidence in control (OF) treatment reached up to $100 \%$ while a lower incidence rate $(29.5 \%)$ of the fumigation (FOF) treatment was observed. Compared with the control treatment, the fumigation followed by biological organic fertilizer (FBOF) treatment significantly decreased the disease incidence to $6.4 \%(P<0.05)$ (Fig. 1$)(93.6 \%$ disease control).

Compared to OF treatment, the population of pathogen decreased significantly in fumigation treatments (FOF and FBOF), pathogens were almost undetectable in these two treatments (Additional file 1: Figure S1).

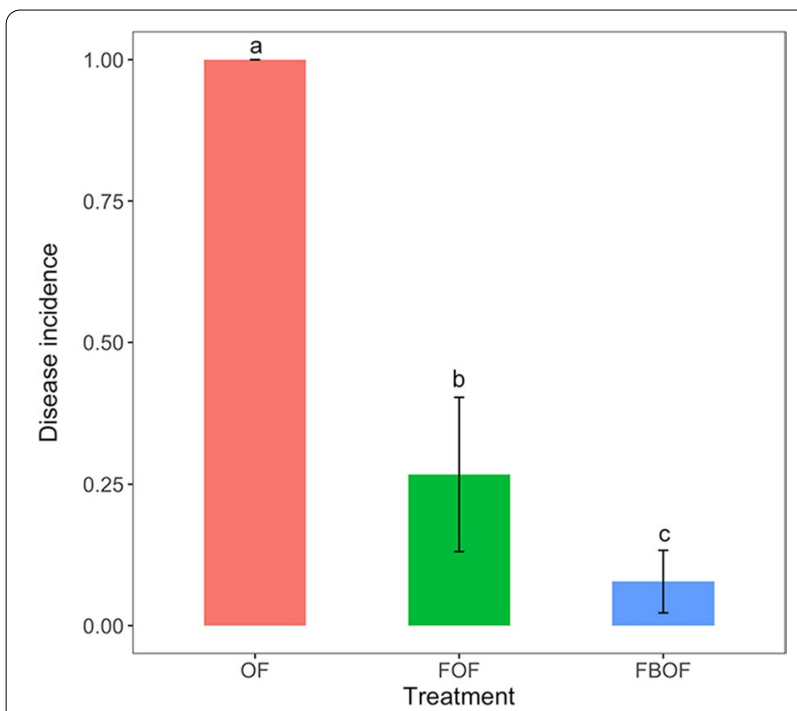

Fig. 1 Disease incidence of watermelon Fusarium wilt in different treatments. OF organic fertilizer, FOF dazomet fumigation followed by organic fertilizer, FBOF dazomet fumigation followed by biological organic fertilizer. Different lowercases indicate a significant difference at the 0.05 probability level according to the Duncan test

\section{Microbial community diversity}

The soil microbial communities (including bacteria and fungi) alpha-diversity (Chao and Shannon) indices in the fumigation (FOF and FBOF) treatments were significantly lower compared with OF treatment. However, there was no significant difference between FOF and FBOF treaments (Table 1).

Nonmetric multidimensional scale (NMDS) analysis based on the Bray-Curtis distance illustrated significant differences in soil microbial community composition between the three treatments (PERMANOVA, $P<0.05$ ) (Fig. 2). Compared to control treatment, the microbial communities in FOF and FBOF treatments were relatively similar.

\section{Microbial community composition}

Under the bacterial phylum level, Proteobacteria were the most abundant, comprising $31.3 \%, 35.3 \%$, and $35.0 \%$ of the bacterial population in the OF, FOF, and FBOF treated soils, respectively (Additional file 1: Figure S2). As observed, the relative abundance of Firmicutes (8.5\%), TM7 (5.7\%), Gemmatimonadetes (5.4\%), and Actinobacteria (5.3\%) in FBOF treatment were significantly higher than OF and FOF treatments. Conversely, the relative abundance of Acidobacteria in FBOF treatment (2.6\%) dramatic lower than that in OF $(7.9 \%)$ and FOF $(4.1 \%)$ treatments.

The relative abundance of the fungi Arthrobotrys (16.5\%) and Phialemonium (8.1\%) in FBOF treatment were significantly higher compared to OF and FOF treatments (Additional file 1: Figure S2). The genus Trichoderma accounted for $2.2 \%, 10.8 \%$ and $13.5 \%$ of the fungal community in the OF, FOF and FBOF treatments, respectively. The relative abundance of Fusarium in OF (7.9\%) was the highest among the three treatments. Compared to OF, the FBOF treatment significantly increased the abundance of Firmicutes (Additional file 1: Figure S3), Bacillus, and Trichoderma (Fig. 3).

Table 1 Soil microbial diversity indices

\begin{tabular}{llrr}
\hline & Treatment & \multicolumn{1}{l}{ Chao } & \multicolumn{1}{l}{ Shannon } \\
\hline Fungi & OF & $2188 \pm 230 \mathrm{a}$ & $6.14 \pm 0.29 \mathrm{a}$ \\
& FOF & $1790 \pm 283 \mathrm{~b}$ & $5.14 \pm 1.04 \mathrm{~b}$ \\
& FBOF & $1782 \pm 461 \mathrm{~b}$ & $4.59 \pm 0.59 \mathrm{~b}$ \\
Bacteria & OF & $6730 \pm 683 \mathrm{a}$ & $11.15 \pm 0.24 \mathrm{a}$ \\
& FOF & $5227 \pm 1135 \mathrm{~b}$ & $10.44 \pm 0.74 \mathrm{~b}$ \\
& FBOF & $5572 \pm 891 \mathrm{~b}$ & $10.30 \pm 0.58 \mathrm{~b}$ \\
\hline
\end{tabular}

Values are means \pm standard deviation $(n=8)$. Different lowercases indicate a significant difference among all treatments $(P<0.05$; Duncan test)

$O F$ organic fertilizer, FOF dazomet fumigation coupled with organic fertilizer, $F B O F$ dazomet fumigation coupled with biological organic fertilizer 

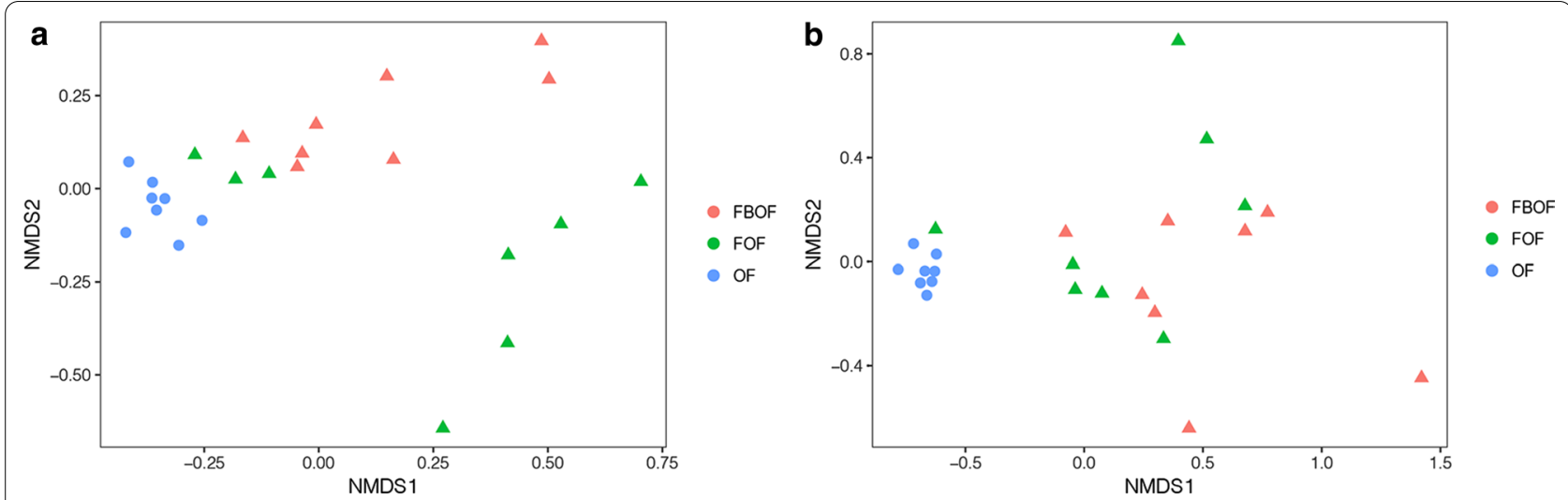

Fig. 2 Nonmetric Multidimensional scaling (NMDS) analysis in bacterial (a) and fungal (b) microbiome between all sample sets. OF organic fertilizer, FOF dazomet fumigation followed by organic fertilizer, FBOF dazomet fumigation followed by biological organic fertilizer
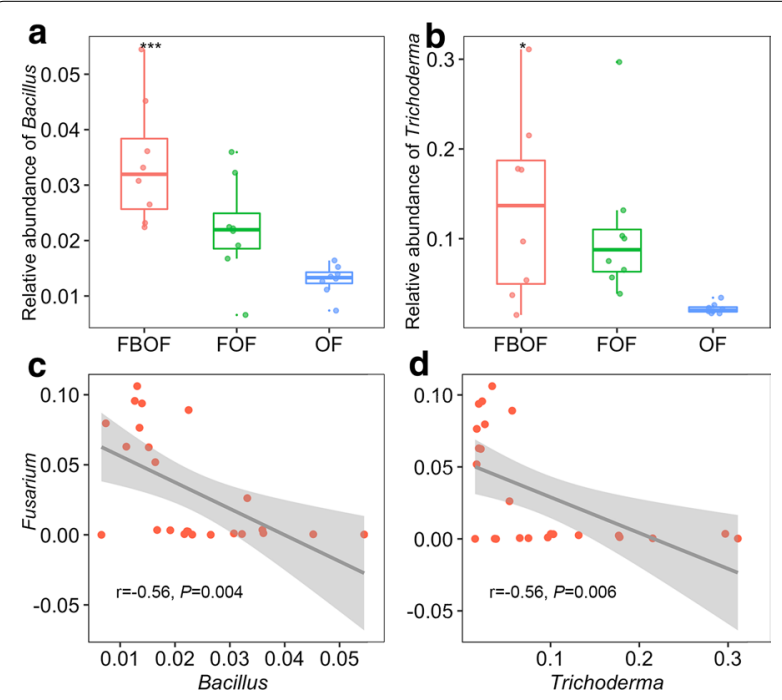

Fig. 3 The relative abundance (RA) of genus Bacillus (a) and

Trichoderma (b) in the three treatments and Pearson correlations ( $r$ ) between RA of Bacillus (c) and Trichoderma (d) with RA of Fusarium. The asterisk indicates significant differences among the treatments, as defined by Duncan test $(P<0.05)$. OF organic fertilizer, FOF dazomet fumigation followed by organic fertilizer, FBOF dazomet fumigation followed by biological organic fertilizer
A Mantel test indicated that the bacterial $(\rho=0.30$, $P<0.01)$ and fungal $(\rho=0.52, P<0.01)$ community compositions were significantly correlated with disease incidence (Additional file 1: Table S1). Among the dominant microbial genera (relative abundance $>1 \%)$, Bacillus $(\mathrm{r}=-0.56, P=0.004)$ and Trichoderma $(\mathrm{r}=-0.56, P=0.006)$ illustrated significant negative correlations with Fusarium relative abundance (Fig. 3).

\section{Different effects of amendments on soil microbial molecular ecological network}

Network plots revealed varying microbial co-occurrence network structures among the three treatments (Fig. 4). Different $\mathrm{R}^{2}$ values of $0.835,0.951$ and 0.918 for FBOF, FOF and OF treatments, respectively, indicating networks formed possess scale-free properties (Additional file 1: Table. S2). With more links in FBOF treatment than FOF treatment, the biological organic fertilizer application after fumigation increased the connectivity, resulting in a more complex soil microbial community.

The topological character of each nodes was demonstrated with Zi-Pi plot (Additional file 1: Figure S4). Majority (96.4\%) of the nodes were peripherals as most of their links inside their modules. About $3.5 \%$ of the nodes were module hubs while only one Bacteroidetes OTU was a connector. Among all the module hubs, a total of 43, 6, and 24 OTUs belonged to the OF, FOF and FBOF treatments, respectively. Three of the 24 FBOF module hubs were Firmicutes, and two of them were closely related to Bacillus. Three module hubs in FBOF were assigned to Ascomycota, of which two were closely related to Trichoderma. The other hubs belonged to varying taxa (i.e., Proteobacteria, Gemmatimonadetes, Chloroflexi, and Acidobacteria) (Additional file 1: Table S3).

\section{Discussion}

As we aimed to explore the underlying mechanisms that link dazomet fumigation followed by biological organic fertilizer application with watermelon Fusarium wilt disease suppression. Two fumigation treatments, especially FBOF (applied biological organic fertilizer after fumigation), were effective in reducing Fusarium wilt disease incidence. This demonstrated that dazomet fumigation was highly effective in suppressing Fusarium wilt disease, 

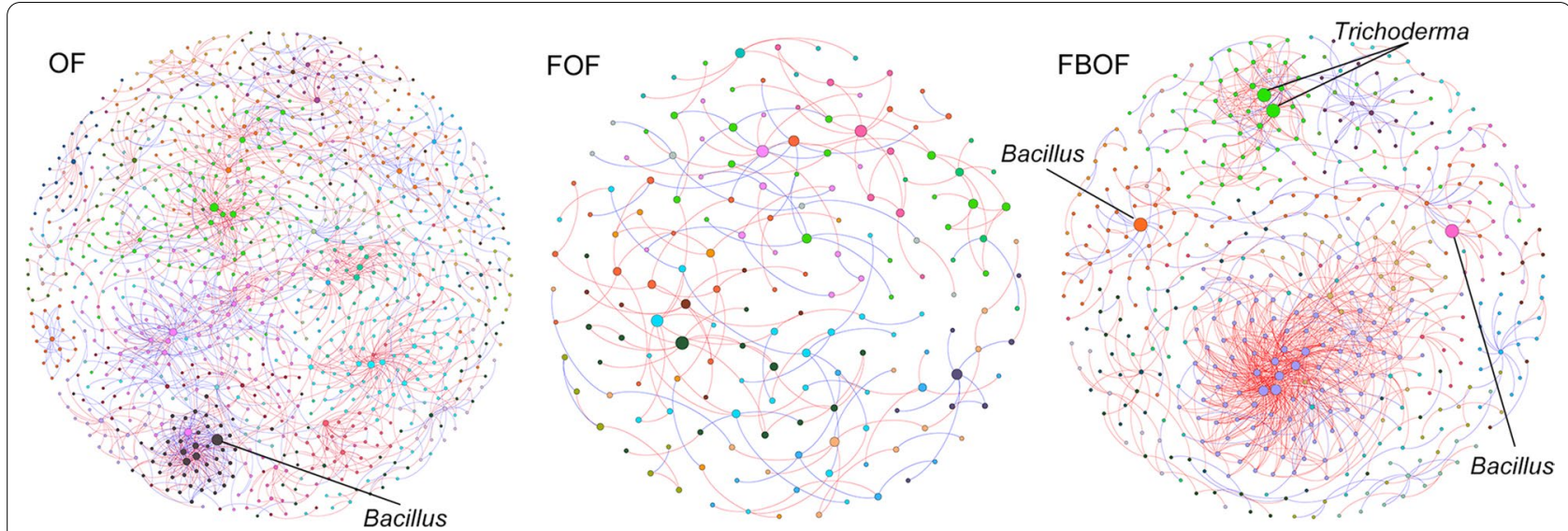

Fig. 4 Different networks performed by OTU tables based on RMT analysis. OF organic fertilizer, FOF dazomet fumigation followed by organic fertilizer, FBOF dazomet fumigation followed by biological organic fertilizer. Different networks represent random matrix theory co-occurrence models for each treatment. Different nodes mean different OTUs, and links between the nodes indicate significant correlation. Modules are randomly colored for, and modules with more than 5 nodes were shown

as supported by previous studies (Tian et al. 2014). In the meantime, treatment with only organic fertilizer (OF) resulted in the highest disease incidence, demonstrating that organic fertilizer application alone was not effective in inducing disease suppression in severe diseased area. A previous study indicated that the application of organic fertilizer alone was ineffective and possibly propitious to the growth of pathogens (Bonanomi et al. 2010). Thus, the utilization of dazomet followed by a biological organic fertilizer amendment was a better alternative for controlling Fusarium wilt disease.

In our greenhouse study, we observed that the microbial communities were principally sensitive to dazomet fumigation and resilient to the disturbance caused by the combination of dazomet fumigation and bio-fertilizer application. In comparison to organic fertilizer amendment alone (OF), both the Chao and Shannon indices exhibited dramatic decreases in microbial richness and diversity in the fumigated treatments. The result is identical with previous findings that fumigation could reduce microbial biodiversity (Fu et al. 2012; Griffiths et al. 2000). The control treatment exhibited significantly higher richness and abundance than the dazomet-treated samples further supporting the impact of fumigation, as dazomet is widely known to suppress the growing of microbial communities (Eo and Park 2014; Scopa and Dumontet 2007). On the basis of the insurance hypothesis, biodiversity loss might lead to a reduction in ecosystem stability (Griffiths and Philippot 2013). Previous experiments have supported the hypothesis by using differential gradient fumigation to decrease soil biodiversity, and the lower biodiversity in soil indicated a reduced resilience in plant decomposition after disturbance (Griffiths et al. 2000).
It seems that the compositional transformation of the intrinsic Fusarium-dominated soil microbial community to a re-assembled non-disease community was the basis for disease suppression (van Elsas et al. 2012). Supporting this speculation, we observed the bacterial and fungal community composition of OF treatment was distinctly different from the other two treatments (FOF, FBOF) which supports the differences in the alpha-diversities between the treatments. This confirms previous results where fumigation often led to diversification in community composition (Ibekwe et al. 2001; Omirou et al. 2010; Shen et al. 2018). In addition, the initial disturbance by fumigation was able to break the stability of soil microbial community by decreasing community diversity and alter the community structure and potentially releasing the available niche space, temporarily decreasing species competition. Then the invader whether colonized in the soil depends on its ability to achieve the available niche space within the disturbed community (Kinnunen et al. 2016).

Two types of fertilizer in our greenhouse study are composts rich $\mathrm{C}, \mathrm{N}$, and exogenous microbes (Bacillus and Trichoderma in bio-fertilizer). When applied to soil, they advance the soil fertility, ameliorate soil structure and increase or decrease several microbial abundances and activities (Ling et al. 2014; Suleiman et al. 2016). Hence, different amendments endowed distinct microbial communities. Lower abundance of the pathogen Fusarium and higher abundances of the antagonistic agents Bacillus and Trichoderma introduced by biological organic fertilizer are likely related to Fusarium wilt disease incidence in this study. Mantel tests revealed that microbial community composition might be a key factor 
in disease suppression. The highest relative abundance of Firmicutes identified within the FBOF treatment revealed that they may be involved in disease suppression in these soils (Trivedi et al. 2017; Xiong et al. 2017b). The Firmicutes include numerous potential biocontrol agents and has been reported with a higher abundance in suppressive soils of different soil-borne disease systems (Rosenzweig et al. 2012; Shen et al. 2015). At the genus level, Bacillus, which was widely used to suppress soil-borne wilt disease, were significant higher in FBOF than other two treatments (Cao et al. 2011; Zhang et al. 2008). Due to their broad-spectrum antibiotic activity and the ability of form endospores, the Bacillus species possess several advantages in excess of other agents for protection against pathogens (Cavaglieri et al. 2005). Previous research on cucurbits showed that fumigation can kill the mycelia of $F$. oxysporum of cucumber and then result in a dramatic abundance diminution of the pathogen ( $\mathrm{Li}$ et al. 2016). For further impact restrained the relative abundance of Fusarium, biological organic fertilizer application builds on the initial express of fumigation, which due to the disease suppressive capacity of microbial populations contained within the biological organic fertilizer. It should be noticed that pathogenic or non-pathogenic Fusarium were not separately quantified in this study yet. Nevertheless, field disease incidence revealed that the pathogenic Fusarium played a main role in the control treatment. Besides, the Fusarium population was very low in the fumigated treatment, so this might not be an issue. As a kind of biological control agent, the genus Trichoderma could compete nutrients and space with pathogens, alter soil conditions, promote the growth of plant, or some direct biocontrol such as mycoparasitism and antibiosis to exert biocontrol against fungal pathogen (Benítez et al. 2004). These beneficial species (Bacillus and Trichoderma) introduced by bio-fertilizer were detected at increased relative abundances in the FBOF treatment. Linear models revealed that higher relative abundance of Bacillus/Trichoderma might generate negative effect on Fusarium relative abundances. Therefore, the colonization of introduced Bacillus and Trichoderma after fumigation in our experiment had a direct effect on pathogen and, consequently, on the suppression of Fusarium wilt disease.

Several previous studies have illustrated that the biological organic fertilizer amendment played a major role in shaping soil microbial community composition (Ling et al. 2014; Qiu et al. 2012). Our results demonstrated that empty niches created due to fumigation are filled by microbial populations introduced by biological organic fertilizer which leads to interactions with the surviving resident populations. In this manner, the remodeling of the soil microbiome, in concert with potential antagonistic capacities, serve to suppress plant disease (Akhtar and Malik 2000).

Microbial diversity contains not only the abundance of species but also the complex interactions among different species (Olesen et al. 2007). Based on ecosystem theory, the sensibility of soil ecosystems to invader rest with their complexity (Fließbach et al. 2009). The use of phylogenetic molecular ecological networks (pMENs) is one of the methods to investigate the complexity of interactions within a microbial community. The fumigated (FOF and FBOF) treatments exhibited a lower number of nodes and links compared with OF treatment, due to the reduced soil microbial community complexity and stability after fumigation. However, compared to the FOF treatment, the FBOF treatment exhibited a higher number of nodes and links and a higher ratio of positive/negative links. Our results indicated that the re-shaped microbial community resulted in more microbial cooperation than competition.

Each soil microbial ecological network is made up of a series of nodes, and each node has a different role in the network topological structure. Although the number of module hubs in fumigation treatments was under non-fumigated treatment, the FBOF treatment contained more module hubs than FOF. This suggests that even though fumigation decreased the overall number of interactions, the biological organic fertilizer amendment resulted in a less drastic decrease in network complexity than organic fertilizer alone. Previous theory predicts that a group of species with higher probability of interacting with each other shows higher resistance and resilience against distribution owing to their buffering of the extinctions (Stouffer and Bascompte 2011). The majority of the module hubs in the FBOF treatment were bacteria, indicating that biological organic fertilizer amendment stimulated the microbial network to recover towards a bacterial- dominant community. Our previous research has demonstrated that Fusarium wilt disease may be more susceptible in fungal- dominant microbial communities (Zhao et al. 2017).

Only three module hubs were identified as fungi. Two of these were identified as the genus Trichoderma and the other one (OTU fun3928) was identified within the order Hypocreaceae. Trichoderma belong to the order Hypocreaceae, thus OTU fun3928 may have similar functions in Fusarium disease suppression as Trichoderma. However, additional research is necessary to support this hypothesis. Two module hubs within the FBOF treatment were assigned to Bacillus. Overall, our results indicate that the introduced species (Bacillus and Trichoderma) within the biological organic fertilizer which were significantly negatively correlated to Fusarium relative abundance, 


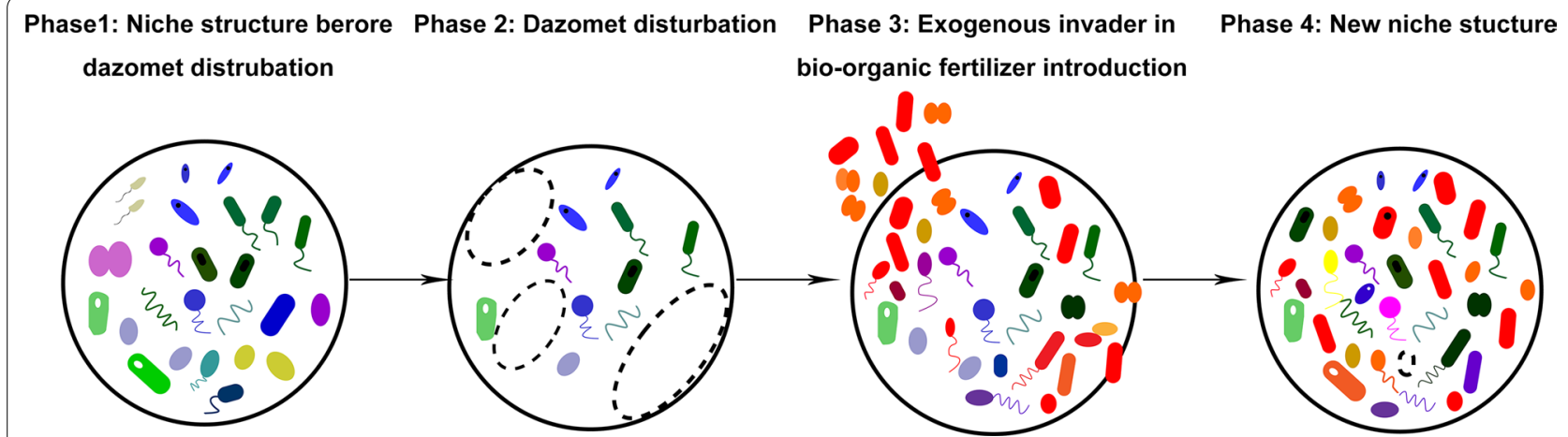

Fig. 5 A conceptual model for understanding fumigation followed by biological organic fertilizer impacts on soil resident microbial community. Consider a microbial community of resident soil as a circle, as shown in (a). When dazomet is applied into the soil, the niches in resident soil are released $(\mathbf{b})$. The introduced species in biological organic fertilizer will take up the niches (c) and alter the niche structure in such way (d)

Dazomet
fumigation
Fig. $\mathbf{6}$ A conceptual cartoon summarizing the influence of dazomet followed by biological organic fertilizer treatments on soil microbial

may be pivotal in the restoration of a suppressive soil microbial community.

A graphical and conceptual interpretation of the mechanism under the observed results is therefore hypothesized (Fig. 5). The initial disturbance by dazomet destroy the competition between numerous microbial taxa, which touches off a competitive release then exogenous species can more easily occupy the free niches. Consequently, the soil microbial community composition is re-arranged, and the niche differentiation pattern of the resident community is altered. This alteration in the community composition with biological organic fertilizer amendment then results in the establishment of a disease-suppressive community that acts through a combination of direct and indirect antagonism towards the pathogen.

In conclusion, the result of this study shows that dazomet fumigation followed by biological organic fertilizer application effectively controlled watermelon Fusarium wilt disease, and is likely attributed to a combination of both the direct suppression of the pathogen population and the re-shaping of the soil microbiome. Fumigation disturbance resulted in a strong reduction in microbial community diversity. This appears to result in niche release and the drastic reduction in interactions within the soil microbial community. The beneficial species (Bacillus and Trichoderma), introduced through biological organic fertilizer amendment re-colonize the vacant niches and, through their interactions, manipulate the composition of the soil microbial community. This promotes a reversion to a relatively complex microbial community. Thus, the overall effectiveness of fumigation followed by biological organic fertilizer application appears to be a combination of introduced microbial direct effects on Fusarium and the promotion of changes in microbial community composition promoted by the biological organic fertilizer (Fig. 6). Therefore, we propose that manipulating the community re-assembly that occurs after fumigation is essential to promote sustainable plant health in the face of a growing worldwide pathogenic threat. 


\section{Supplementary Information}

The online version contains supplementary material available at https://doi. org/10.1186/s13568-021-01225-5.

Additional file 1: Table S1. Spearman's correlations based on Bray-Curtis distance between Fusarium wilt disease incidence and microbial community composition determined by Mantel test. Table S2. Topological properties of the empirical phylogenetic molecular ecological networks (pMENs) among different treatments in comparison to the random networks. Table S3. Phylogenetic relationships of special OTUs in Zi-Pi plot of bulk and rhizosphere soil. Figure S1. Effect of different treatments on the population of pathogens. OF: Organic fertilizer, FOF: Dazomet fumigation coupled with organic fertilizer, FBOF: Dazomet fumigation coupled with bio-organic fertilizer. Different letters indicate significant differences among the treatments, as defined by Duncan test $(P<0.05)$.

Figure S2. The relative abundance of bacterial phyla (A) and fungal genus (B) in the three treatments. OF: Organic fertilizer, FOF: Dazomet fumigation coupled with organic fertilizer, FBOF: Dazomet fumigation coupled with bio-organic fertilizer. Figure $\mathbf{S 3}$. Manhattan plots showing soil enriched OTUs in bacterial (A) and fungal (B) microbial communities between FBOF and OF treatment. OF: Organic fertilizer, FBOF: Dazomet fumigation coupled with bio-organic fertilizer. The dashed line corresponds to the false discovery rate-corrected $P$ value threshold of significance $(a=0.05)$. The color of each dot represents the different taxonomic affiliation of the OTUs (phyla level), and the size corresponds to their RAs in the respective samples. Figure S4. Zi-Pi plot showing the distribution of OTUs based on their topological roles. OF: Organic fertilizer, FOF: Dazomet fumigation coupled with organic fertilizer, FBOF: Dazomet fumigation coupled with bio-organic fertilizer. Each symbol represents an OTU in network. The threshold values of $\mathrm{Zi}$ and Pi for categorizing OTUs were 2.5 and 0.62 , respectively.

\section{Acknowledgements}

The authors would like to acknowledge the support of the National Key Research and Development Program (2017YFD0200805), the National Natural Science Foundation of China (31902113, 31772398), the China Postdoctoral Science Foundation (2017M621761 and 2018T110510), the Priority Academic Program Development of Jiangsu Higher Education Institutions (PAPD), and the Fundamental Research Funds for the Central Universities (KJQN202018). We also would like to express our sincere gratitude to three anonymous reviewers for their valuable comments, which have greatly improved this paper.

\section{Authors' contributions}

This study was conceived and led by QS. XZ, DF, XH and MW did the field experiment and collected the samples. XZ, CZ and JJ finished all the lab work. $X Z$ and $C X$ analyzed the data and wrote the manuscript. All authors discussed the results and their implications and commented on the manuscript as it progressed. All authors read and approved the final manuscript.

\section{Funding}

This study was supported by the National Key Research and Development Program (2017YFD0200805), the National Natural Science Foundation of China $(31902113,31772398)$, the China Postdoctoral Science Foundation (2017M621761 and 2018T110510), the Priority Academic Program Development of Jiangsu Higher Education Institutions (PAPD), and the Fundamental Research Funds for the Central Universities (KJQN202018).

\section{Availability of data and materials}

All data generated or analyzed during this study are included in this published article (and its Additional file).

\section{Declarations}

Ethics approval and consent to participate

Not applicable.

\section{Consent for publication}

Not applicable.

\section{Competing interests}

The authors declare no competing non-financial/financial interests.

Received: 2 March 2021 Accepted: 28 April 2021

Published online: 12 June 2021

\section{References}

Akhtar M, Malik A (2000) Roles of organic soil amendments and soil organisms in the biological control of plant-parasitic nematodes: a review. Biores Technol 74:35-47

Allison SD, Martiny JBH (2008) Resistance, resilience, and redundancy in microbial communities. Proc Natl Acad Sci 105:11512-11519

Bailey KL, Lazarovits G (2003) Suppressing soil-borne diseases with residue management and organic amendments. Soil Tillage Res 72:169-180

Benítez T, Rincón AM, Limón MC, Codón AC (2004) Biocontrol mechanisms of Trichoderma strains. Int Microbiol 7:249-260

Bonanomi G, Antignani V, Capodilupo M, Scala F (2010) Identifying the characteristics of organic soil amendments that suppress soilborne plant diseases. Soil Biol Biochem 42:136-144

Cao Y, Zhang Z, Ling N, Yuan Y, Zheng X, Shen B, Shen Q (2011) Bacillus subtilis SQR 9 can control Fusarium wilt in cucumber by colonizing plant roots. Biol Fertil Soils 47:495-506

Caporaso JG, Kuczynski J, Stombaugh J, Bittinger K, Bushman FD, Costello EK Fierer N, Peña AG, Goodrich JK, Gordon Jl, Huttley GA, Kelley ST, Knights D, Koenig JE, Ley RE, Lozupone CA, McDonald D, Muegge BD, Pirrung M, Reeder J, Sevinsky JR, Turnbaugh PJ, Walters WA, Widmann J, Yatsunenko T, Zaneveld J, Knight R (2010) QIIME allows analysis of high-throughput community sequencing data. Nat Methods 7:335-336

Cavaglieri L, Orlando J, Rodríguez MI, Chulze S, Etcheverry M (2005) Biocontrol of Bacillus subtilis against Fusarium verticillioides in vitro and at the maize root level. Res Microbiol 156:748-754

Deng Y, Jiang YH, Yang Y, He Z, Luo F, Zhou J (2012) Molecular ecological network analyses. BMC Bioinform 13:113-121

Eo J, Park KC (2014) Effects of dazomet on soil organisms and recolonisation of fumigated soil. Pedobiologia 57:147-154

Fließbach A, Winkler M, Lutz MP, Oberholzer HR, Mäder P (2009) Soil amendment with Pseudomonas fluorescens CHAO: lasting effects on soil biological properties in soils low in microbial biomass and activity. Microb Ecol 57:611-623

Fu CH, Hu BY, Chang TT, Hsueh KL, Hsu WT (2012) Evaluation of dazomet as fumigant for the control of brown root rot disease. Pest Manag Sci 68:959-962

Fuente La, De L, Landa BB, Weller DM (2006) Host crop affects rhizosphere colonization and competitiveness of 2,4-diacetylphloroglucinol-producing Pseudomonas fluorescens. Phytopathology 96:751-762

Fujino C, Wada S, Konoike T, Toyota K, Suga Y, Ikeda JI (2008) Effect of different organic amendments on the resistance and resilience of the organic matter decomposing ability of soil and the role of aggregated soil structure. Soil Sci Plant Nutr 54:534-542

Griffiths BS, Philippot L (2013) Insights into the resistance and resilience of the soil microbial community. FEMS Microbiol Rev 37:112-129

Griffiths BS, Ritz K, Bardgett RD, Cook R, Christensen S, Ekelund F, Sorensen SJ, Baath E, Bloem J, de Ruiter PC, Dolfing J, Nicolardot B (2000) Ecosystem response of pasture soil communities to fumigation-induced microbial diversity reductions: an examination of the biodiversity-ecosystem function relationship. Oikos 90:279-294

Ibekwe AM, Papiernik SK, Gan J, Yates SR, Yang CH, Crowley DE (2001) Impact of fumigants on soil microbial communities. Appl Environ Microbiol 67:3245-3257

Kinnunen M, Dechesne A, Proctor C, Hammes F, Johnson D, Quintela-Baluja M, Graham D, Daffonchio D, Fodelianakis S, Hahn N, Boon N, Smets BF (2016) A conceptual framework for invasion in microbial communities. ISME J 10:2773-2775

Komada H (1975) Development of a selective medium for quantitative isolation of Fusarium oxysporum from natural soil. Rev Plant Prot Res 8:114-124 
Li R, Shen Z, Sun L, Zhang R, Fu L, Deng X, Shen Q (2016) Novel soil fumigation method for suppressing cucumber Fusarium wilt disease associated with soil microflora alterations. Appl Soil Ecol 101:28-36

Ling N, Huang Q, Guo S, Shen Q (2010a) Paenibacillus polymyxa SQR-21 systemically affects root exudates of watermelon to decrease the conidial germination of Fusarium oxysporum f. sp. niveum. Plant Soil 341:485-493

Ling N, Xue C, Huang Q, Yang X, Xu Y, Shen Q (2010b) Development of a mode of application of bioorganic fertilizer for improving the biocontrol efficacy to Fusarium wilt. Biocontrol 55:673-683

Ling N, Deng K, Song Y, Wu Y, Zhao J, Raza W, Huang Q, Shen Q (2014) Variation of rhizosphere bacterial community in watermelon continuous monocropping soil by long-term application of a novel bioorganic fertilizer. Microbiol Res 169:570-578

Ling N, Zhu C, Xue C, Chen H, Duan Y, Peng C, Guo S, Shen Q (2016) Insight into how organic amendments can shape the soil microbiome in longterm field experiments as revealed by network analysis. Soil Biol Biochem 99:137-149

Luo J, Ran W, Hu J, Yang X, Xu Y, Shen Q (2010) Application of bio-organic fertilizer significantly affected fungal diversity of soils. Soil Sci Soc Am J 74:2039-2110

Olesen JM, Bascompte J, Dupont YL, Jordano P (2007) The modularity of pollination networks. Proc Natl Acad Sci USA 104:19891-19896

Omirou M, Rousidou C, Bekris F, Papadopoulou KK, Menkissoglou-Spiroudi U, Ehaliotis C, Karpouzas DG (2010) The impact of biofumigation and chemical fumigation methods on the structure and function of the soil microbial community. Microb Ecol 61:201-213

Qiu M, Zhang R, Xue C, Zhang S, Li S, Zhang N, Shen Q (2012) Application of bioorganic fertilizer can control Fusarium wilt of cucumber plants by regulating microbial community of rhizosphere soil. Biol Fertil Soils 48:807-816

Rosenzweig N, Tiedje JM, Quensen JF, Meng Q, Hao JJ (2012) Microbial communities associated with potato common scab-suppressive soil determined by pyrosequencing analyses. Plant Dis 96:718-725

Rykiel EJ (1985) Towards a definition of ecological disturbance. Aust J Ecol $10: 361-365$

Saeed IAM, Rouse DI, Harkin JM, Smith KP (2007) Effects of soil water content and soil temperature on efficacy of metham-sodium against Verticillium dahliae. Plant Dis 81:773-776

Scopa A, Dumontet S (2007) Soil solarization: effects on soil microbiological parameters. J Plant Nutr 30:537-547

Shen Z, Ruan Y, Wang B, Zhong S, Su L, Li R, Shen Q (2015) Effect of biofertilizer for suppressing Fusarium wilt disease of banana as well as enhancing microbial and chemical properties of soil under greenhouse trial. App Soil Ecol 93:111-119

Shen Z, Xue C, Penton CR, Thomashow LS, Zhang N, Wang B, Ruan Y, Li R, Shen Q (2018) Suppression of banana Panama disease induced by soil microbiome reconstruction through an integrated agricultural strategy. Soil Biol Biochem 128:164-174

Slusarski C, Pietr SJ (2009) Combined application of dazomet and Trichoderma asperellum as an efficient alternative to methyl bromide in controlling the soil-borne disease complex of bell pepper. Crop Prot 28:668-674

Stouffer DB, Bascompte J (2011) Compartmentalization increases food-web persistence. Proc Natl Acad Sci USA 108:3648-3652

Suleiman AKA, Gonzatto R, Aita C, Lupatini M, Jacques RJS, Kuramae EE, Antoniolli Zl, Roesch LFW (2016) Temporal variability of soil microbial communities after application of dicyandiamide-treated swine slurry and mineral fertilizers. Soil Biol Biochem 97:71-82

Tian T, Li SD, Sun MH (2014) Synergistic effect of dazomet soil fumigation and Clonostachys rosea against cucumber Fusarium wilt. Phytopathology 104:1314-1321

Trivedi P, Delgado-Baquerizo M, Trivedi C, Hamonts K, Anderson IC, Singh BK (2017) Keystone microbial taxa regulate the invasion of a fungal pathogen in agro-ecosystems. Soil Biol Biochem 111:10-14

Van Elsas JD, Chiurazzi M, Mallon CA, Elhottova D, Kristufek V, Salles JF (2012) Microbial diversity determines the invasion of soil by a bacterial pathogen. Proc Natl Acad Sci USA 109:1159-1164

Wertz S, Degrange V, Prosser J, Poly F, Commeaux C, Guillaumaud N, Le Roux X (2007) Decline of soil microbial diversity does not influence the resistance and resilience of key soil microbial functional groups following a model disturbance. Environ Microbiol 9:2211-2219

White TJ, Bruns T, Lee S, Taylor J (1990) Amplification and direct sequencing of fungal ribosomal RNA genes for phylogenetics. PCR Protoc 18:315-322

Wu K, Yuan S, Wang L, Shi J, Zhao J, Shen B, Shen Q (2014) Effects of bio-organic fertilizer plus soil amendment on the control of tobacco bacterial wilt and composition of soil bacterial communities. Biol Fertil Soils 50:961-971

Xiong W, Guo S, Jousset A, Zhao Q, Wu H, Li R, Kowalchuk GA, Shen Q (2017a) Bio-fertilizer application induces soil suppressiveness against Fusarium wilt disease by reshaping the soil microbiome. Soil Biol Biochem 114:238-247

Xiong W, Li R, Ren Y, Liu C, Zhao Q, Wu H, Jousset A, Shen Q (2017b) Distinct roles for soil fungal and bacterial communities associated with the suppression of vanilla Fusarium wilt disease. Soil Biol Biochem 107:198-207

Xu N, Tan G, Wang H, Gai X (2016) Effect of biochar additions to soil on nitrogen leaching, microbial biomass and bacterial community structure. Eur J Soil Biol 74:1-8

Yuan S, Li M, Fang Z, Liu Y, Shi W, Pan B, Wu K, Shi J, Shen B, Shen Q (2016) Biological control of tobacco bacterial wilt using Trichoderma harzianum amended bioorganic fertilizer and the arbuscular mycorrhizal fungi Glomus mosseae. Biol Control 92:164-171

Zarraonaindia I, Owens SM, Weisenhorn P, West K, Hampton-Marcell J, Lax S, Bokulich NA, Mills DA, Martin G, Taghavi S, van der Lelie D, Gilbert JA (2015) The soil microbiome influences grapevine-associated microbiota. Mbio 6:e02527-e2614

Zhang S, Raza W, Yang X, Hu J, Huang Q, Xu Y, Liu X, Ran W, Shen Q (2008) Control of Fusarium wilt disease of cucumber plants with the application of a bioorganic fertilizer. Biol Fertil Soils 44:1073-1080

Zhao J, Mei Z, Zhang X, Xue C, Zhang C, Ma T, Zhang S (2017) Suppression of Fusarium wilt of cucumber by ammonia gas fumigation via reduction of Fusarium population in the field. Sci Rep 7:43103-43109

Zhou XG, Everts KL (2004) Suppression of Fusarium wilt of watermelon by soil amendment with hairy vetch. Plant Dis 88:1357-1365

Zhou J, Deng Y, Luo F, He Z, Tu Q, Zhi X (2010) Functional molecular ecological networks. mBio 1:e00169-10-e00169-19

Zhou J, Deng Y, Luo F, He Z, Yang Y (2011) Phylogenetic molecular ecological network of soil microbial communities in response to elevated $\mathrm{CO}_{2}$. mBio 2:e00122-11

\section{Publisher's Note}

Springer Nature remains neutral with regard to jurisdictional claims in published maps and institutional affiliations. 